\title{
Preliminary study of thrombogenicity induced by the nanoparticle surface coating of intracranial stents
}

\author{
A. Chiriac ${ }^{1}$, Georgiana Ion ${ }^{1}$, G. Stan ${ }^{2}$, T. Popescu ${ }^{2}$, \\ Mihaela Sofronie $^{2}$, I. Poeata ${ }^{1}$ \\ 1 "Gr. T. Popa" University of Medicine and Pharmacy, lasi, RoMANIA \\ 2 National Institute of Materials Physics, Bucharest-Magurele, ROMANIA
}

\begin{abstract}
Endovascular treatment of intracranial aneurysms with intracranial stents was proven to be clinically safe and effective, but is still associated with a risk of thromboembolic complications. Stent thrombosis could be a sever complication associated with specific stent surface coatings and designs. Standardized in vitro tests for investigation of thrombogenicity induced by different nanomaterials were used as the basic method in carrying out the present study. Therefore, the aim of this study was to evaluate the thrombogenicity of three different nanomaterials ( $\mathrm{ZnO}, \mathrm{TiO} 2 \mathrm{si}$ $\mathrm{Fe} 3 \mathrm{O} 4$ ) possible used as surface coating for intracranial stents. This study is based on a procedure for in vitro analyses of plasma coagulation time. To measure the plasma coagulation time, platelet-poor plasma from human whole blood was in vitro exposed to nanoparticles and analysed in prothrombin (PT) and activated partial thromboplastin (APTT).
\end{abstract}

\section{INTRODUCTION}

A variety of intracranial stents are used in permanent blood contact. Thrombogenicity is the property of a material to induce a thrombus formation, which may result in partial or complete blood vessel occlusion. Thus, thrombogenicity of intracranial stent may have important implication in the long-term outcome of neurosurgical patients that may lead to a life-threatening condition such as stroke. Stent / blood contact surface activity has been a widely subject discussed and reported and, notwithstanding the clinical significance of this phenomenon is still controversial.

Moreover, the surface coatings of these implants that are in permanent contact with blood are often subjected to tests on coagulation in order to use the least thrombogenic materials. Activated partial thromboplastin time (aPTT), prothrombin time (PT) and the international normalized ratio (INR) are indicated as the techniques of choice used to help detect and diagnose a bleeding disorder or excessive clotting disorder. In our study, an in vitro model was established, which allows investigation of neurovascular stents with

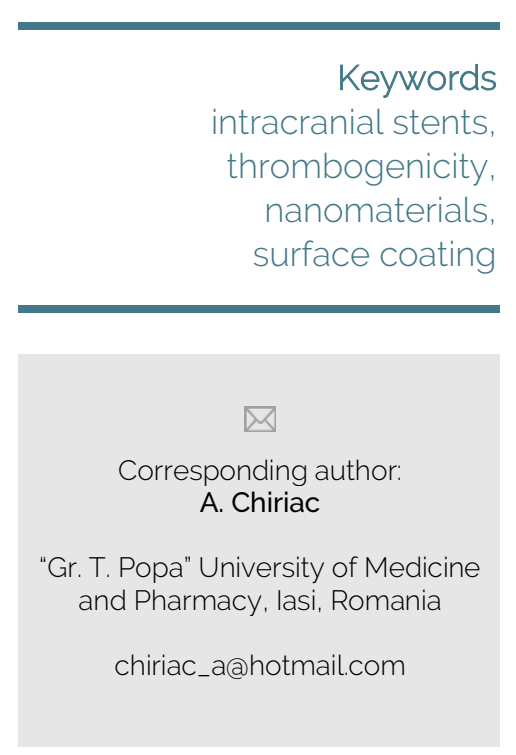

Copyright and usage. This is an Open Access article, distributed under the terms of the Creative commons Attribution Non-Commercial No Derivatives License (https://creativecommons org/licenses/by-nc-nd/4.0/) which permits noncommercial re-use, distribution, and reproduction in any medium, provided the original work is unaltered and is properly cited

The written permission of the Romanian Society of Neurosurgery must be obtained for commercial re-use or in order to create a derivative work.

ISSN online 2344-4959 (C) Romanian Society of Neurosurgery

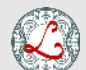

First published ondon Academic Publishing www.lapub.co.uk 
regard to thrombogenicity and coagulation activation. The preliminary study was designed to compare these 3 methods to address the coagulation property of three biomaterials ( $\mathrm{ZnO}$, $\mathrm{TiO} 2$ si $\mathrm{Fe} 3 \mathrm{O} 4$ ) with emphasis on the sensitivity and reproducibility of the test. These three biomaterials prepared in the form of nanoparticles were evaluated for their coagulation profile during incubation in human plasma. The study presents a revised version of these methods to include updated details on sample preparation of nanoparticles, selection of nanoparticle concentration for in vitro study, and updated details on test controls $[4,5,6]$.

\section{MATERIALS AND METHODS}

Platelet rich plasma (PRP) is obtained from fresh human blood derivative incubated with nanoparticles for 15 minutes at $37^{\circ} \mathrm{C}$. This probe is subsequently analysed by evaluating the plasma coagulation time and how the nanoparticles affect this part of the haemostasis control system.

The materials listed below are required for coagulation tests for the study of nanoparticles (Fig. 1):

1. Vacuum test tubes for coagulation testing;

2. $12 \%$ sodium citrate.

3. Thermostat bath;

4. Special centrifuge with tubes test stand;

5. Fresh human blood completely anti-coagulated with sodium citrate and obtained from at least three healthy donors known to be out of any antiinflammatory and antihistamine drug, blood thinning agents and birth control pills;

6. APTT and PT / INR test cards for Abrazo Cascade equipment;

7. Coagulation tester analyzer Abrazo Cascade System from Helena Laboratories;

8. Nanoparticle sample for analysis.

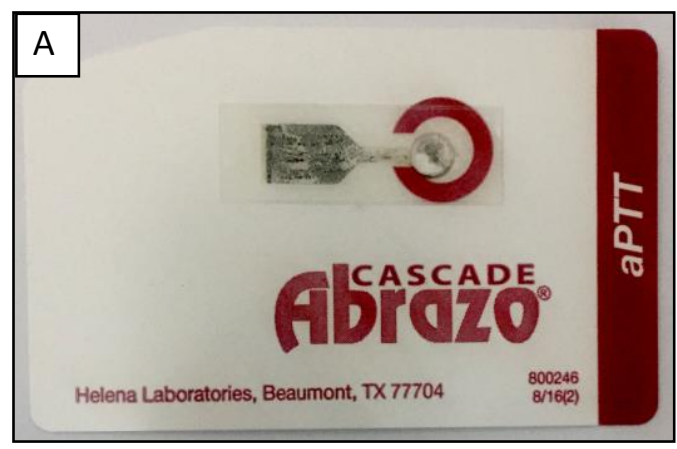

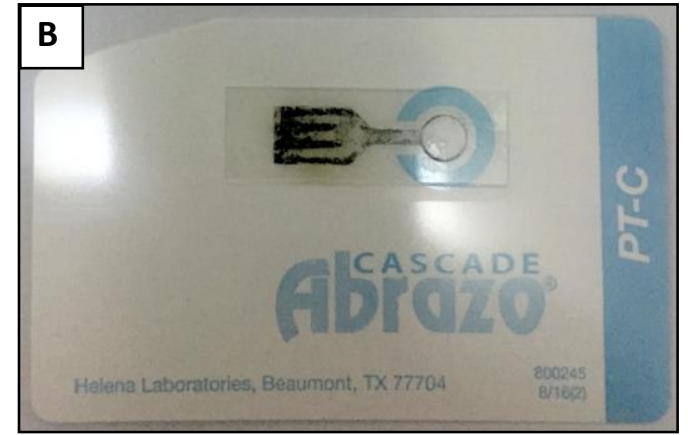
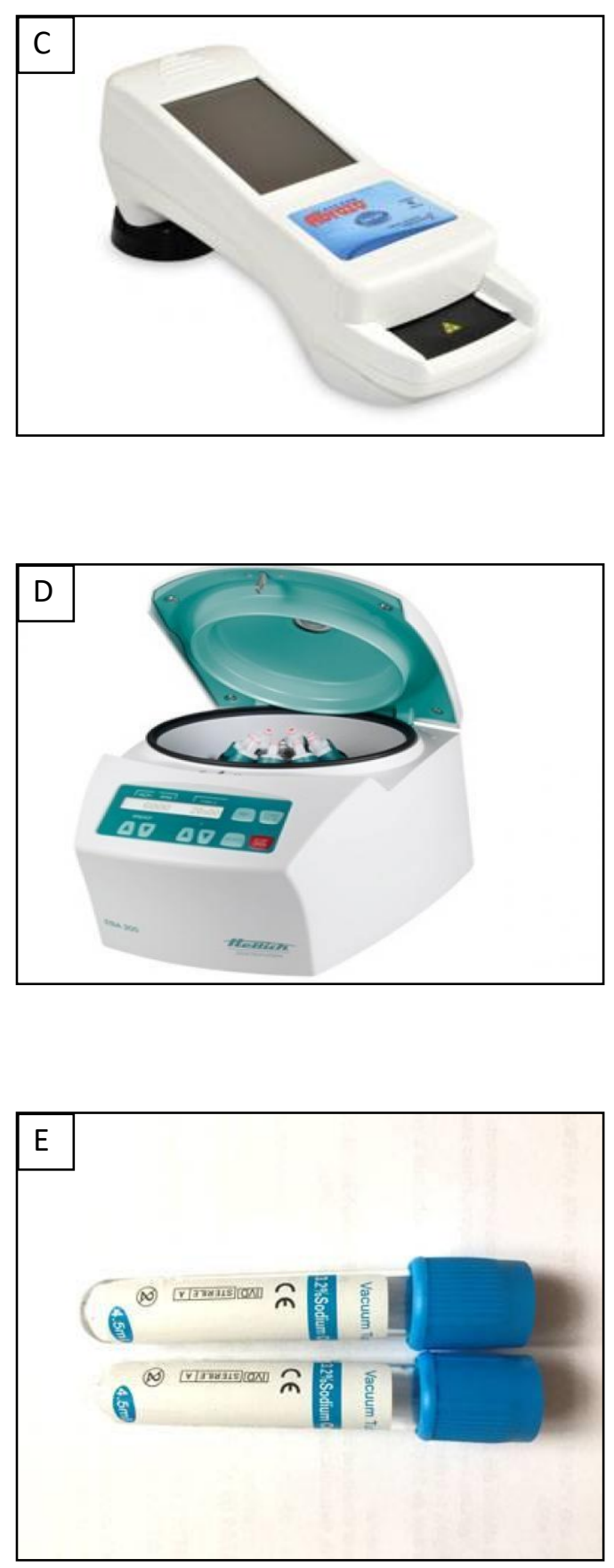


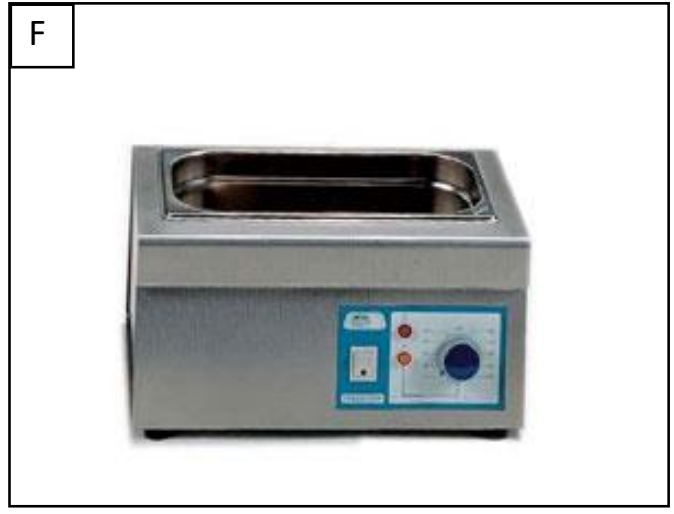

Figure 1: A- aPTT tester card; B- PT / INR tester card; C- Abrazo Cascade Coagulation Test Equipment; D- Centrifuge; EVacuum test tubes with citrate; F- Thermostatic bath.

\section{PREPARATION OF THE STUDY SAMPLES}

The concentrations used in these assays are based on an estimated plasma concentration in a mediumsized human patient at the desired therapeutic dose. In our study, we refer to this concentration as the theoretical concentration of plasma. The hypotheses and considerations for estimating theoretical plasma concentration have been reviewed in the literature
[4] and are summarized below.

For the calculation of nanoparticle concentration, we used in vitro test reports in mice.

If the mouse dose used is approximately $123 \mathrm{mg} / \mathrm{Kg}$ in this example the human dose can be calculated as follows:

$$
\text { human dose }=\frac{\text { mouse dose }}{12.3}=\frac{123 \frac{\mathrm{mg}}{\mathrm{kg}}}{12.3}=10 \mathrm{mg} / \mathrm{kg}
$$

It is known that blood volume accounts for about 8\% of body weight (for example, an adult of $70 \mathrm{~kg}$ has about $5.6 \mathrm{~L}$ ( $8 \%$ of 70 ) blood. This allows an extremely crude estimate of maximum blood concentration.

$$
\begin{aligned}
& \text { in vitro concentration } \begin{aligned}
& \text { human } \text { matrix }=\frac{\text { human dose }}{\text { human blood volume }}=\frac{70 \mathrm{~kg} \times 10 \frac{\mathrm{mg}}{\mathrm{kg}}}{5.6 \mathrm{~L}} \\
&=\frac{700 \mathrm{mg}}{5.6 \mathrm{~L}}=0.125 \mathrm{mg} / \mathrm{mL}
\end{aligned}
\end{aligned}
$$

Each test evaluates a nanoparticle formulation of three different materials at preset concentrations. When the predicted therapeutic concentration is not known, the highest final concentration is $1 \mathrm{mg}$ per $\mathrm{ml}$.
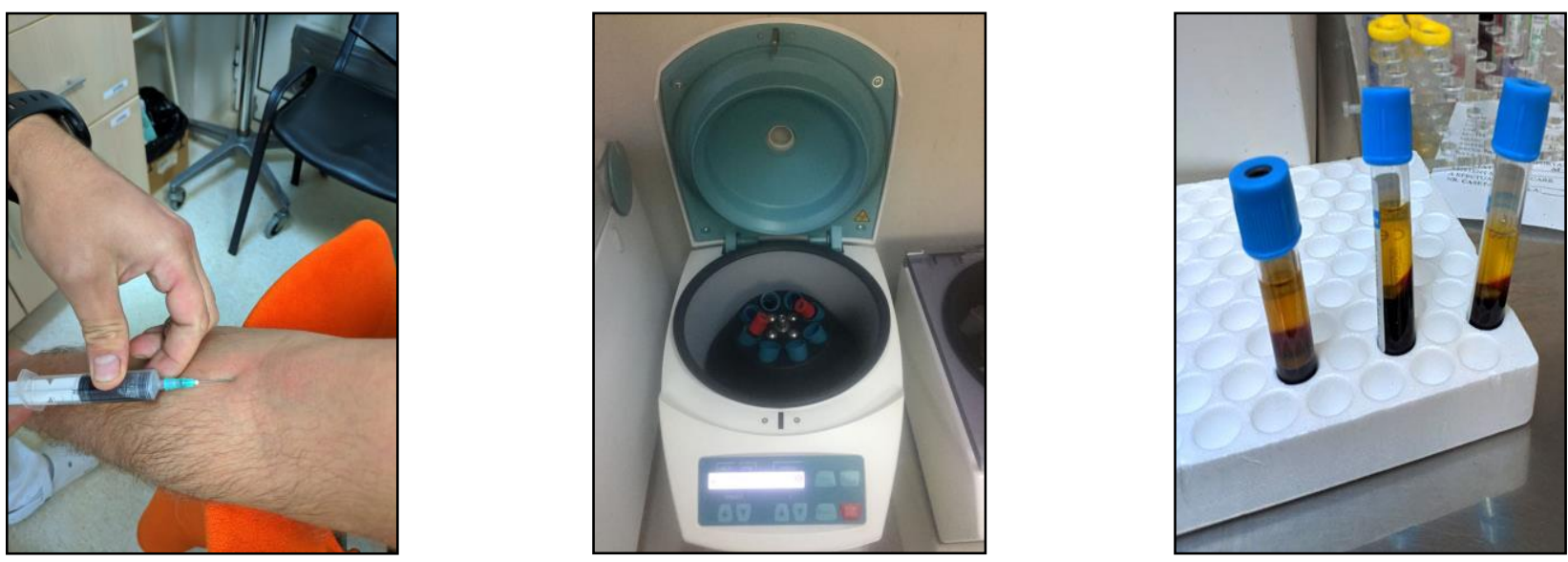

Figure 2: A- Blood collection; B- Centrifuge with multiple cartridges; C- Vacutainer tubes with centrifuged plasma.

\section{PLASMA PREPARATION}

When the blood collection procedure is started the first $10 \mathrm{ml}$ of blood must be discarded [fig.2] due to necessity to prevent the activation of cells and plasma biochemical cascades by the venous puncture procedure. Exposure and storage of blood or plasma at extreme temperatures ( $<20 \mathrm{C}$ or $>37 \mathrm{C}$ ) can affect the quality of test results and should be avoided. Two types of plasma can be obtained to perform this experiment: platelet rich plasma (PRP), platelet poor plasma (PPP). Plasma from individual donors can be analyzed separately or collected together. The combined plasma is prepared by mixing plasma from at least two individual donors. 


\section{NORMAL TEST PLASMA PREPARATION}

Fresh whole blood must be used within 1 hour after collection. The blood is then centrifuged for $10 \mathrm{~min}$ at $3000 \mathrm{~g}$ at $20-22 \mathrm{C}$ and plasma is collected from at least two donors. The combined plasma is stable for 8 hours at room temperature. The assay can also be performed in plasma from individual donors, when needed for mechanical tracking experiments. Two duplicates (four total samples) of plasma test are analyzed in each of the coagulation tests. A duplicate is performed prior to analyzing the nanoparticle samples and the second duplicate at the end of each run to verify that plasma functionality is not affected throughout the experiment.

\section{NANOPARTICLE-TREATED TEST PLASMA PREPARATION}

In a micro-tube, $0.125 \mathrm{mg}$ of nanoparticles and $1 \mathrm{ml}$ of plasma are combined. Mix well on a shaker and incubate for $30 \mathrm{~min}$ at $37^{\circ} \mathrm{C}$. Three test tubes are prepared for each test sample (ie, when each nanoparticle is tested at four concentrations, one needs three test tubes for each concentration for a total of 12 test tubes per nanoparticle test).

\section{PLASMA COAGULATION TESTS (Fig. 3)}

1. Prepare the Cascade Abrazo equipment as described in the manufacturer's manual.

2. Preheat all tubes to $37 \mathrm{C}$.

3. Configure the instrument test parameters for each of the two tests (APTT and PT / INR).

4. Add to the micro-tubes with pre-loaded nanoparticles $1 \mathrm{ml}$ of plasma.

5. Stir micro-tubes for 5 minutes.

6. Scan each specific tester card (aPTT and INR / PT) before testing;

7. Insert the test card into the Cascade Abrazo device;

8. Allow the instrument to warm up before use.

9. Extract the test solution with the micropipette.

10. When the test timer starts, transfer the solution to the test card's geode by pressing the PIP button to activate the pipette.

11. When the time is up, the device will beep and the recording of coagulation time will remain displayed on the screen until further testing begins.

12. The average of the three values must be calculated for each control or test.

13. The average for each control and test sample should be between $25 \%$.

14. If two duplicates of the same study sample showed different results more than $5 \%$, this sample was reanalysed.

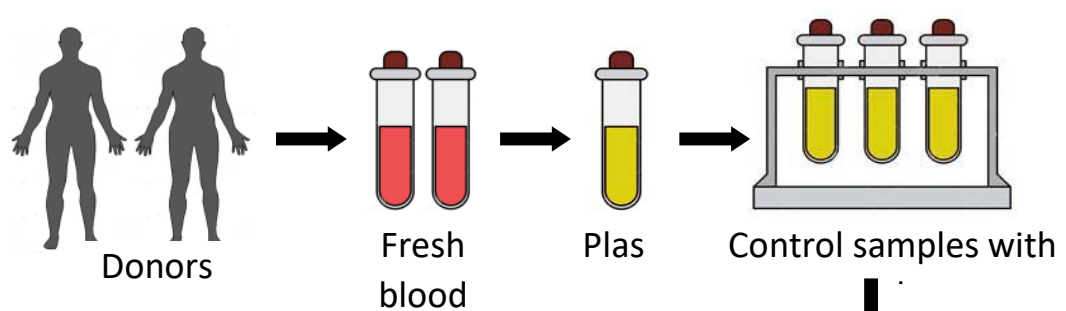

blood

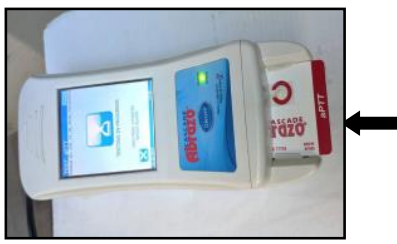

Card insertion

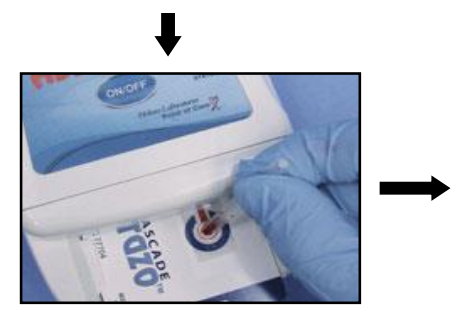

Add test

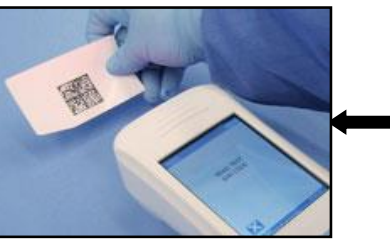

Card scan

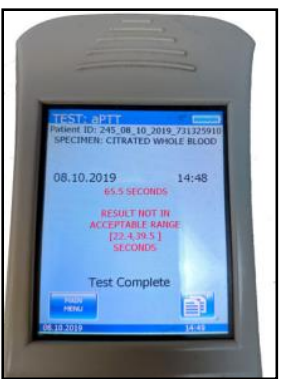

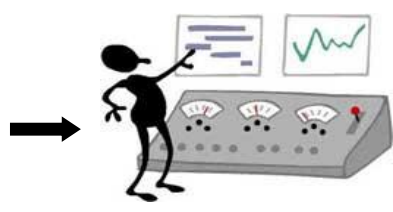

Data recording and analysis
Figure 3: Schematic diagram of coagulation time measurement. 


\section{RESULTS}

The results shown in this study indicate that $\mathrm{ZnO}$ nanoparticles administered in the blood vessels can adsorb the coagulation factors in a specific form to the surface functional group, without activating these factors and that the adsorbed coagulation factors are not functional. Also, $\mathrm{ZnO}$ nanoparticles delayed the coagulation time with the size or surface functional group specificity, because all types of $\mathrm{ZnO}$ nanoparticles adsorbed the coagulation factors of the common pathway, some types of nanoparticles adsorbing more factors than others. The thrombogenicity study of $\mathrm{TiO}_{2}$ and $\mathrm{Fe} 3 \mathrm{O} 4$ nanoparticles suggests that nanoparticles should be an effective agent in prolonging the blood clotting time, thus exerting an anti-coagulant effect, thus improving the hemocompatibility of the coating. The ability of $\mathrm{TiO}_{2}$ and $\mathrm{Fe} 3 \mathrm{O} 4$ nanoparticles to regulate platelet adhesion and plasma coagulation was thus demonstrated in vitro.

\section{PT COAGULATION TEST FOR NANOPARTICLES}

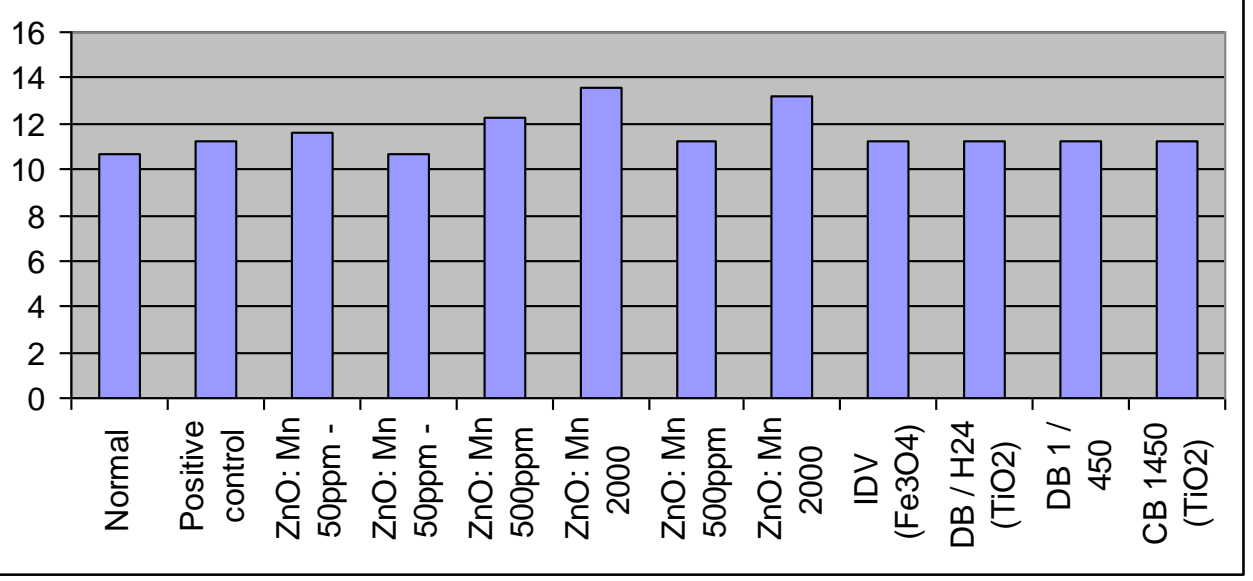

\section{aPPT COAGULATION TEST FOR NANOPARTICLES}

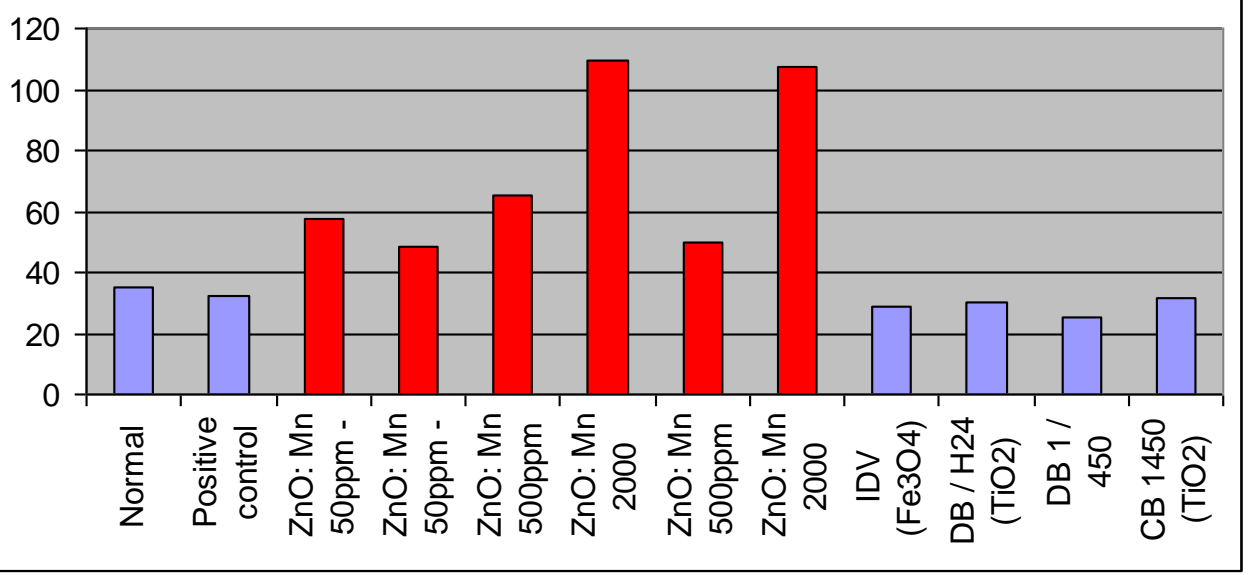

\section{DISCUSSIONS}

Treatment of neurosurgical patients with functionalized coating surfaces stents continues to evolve with the current emergence of nanoparticle functionalization technology that offers a combination of pharmacological and mechanical approaches to prevent thrombosis and arterial restenosis. Despite the promising short term and mid-term outcomes of surface coated stents, there are serious concerns about adverse clinical effects of late stent thrombosis. Certain nanoparticles intended for drug delivery application are designed 
to reduce the effect of thrombogenicity of an intracranial stent in the bloodstream for a prolonged coagulation time in the circulatory system. Thus, a larger therapeutic window is offered until systemic anti-aggregation is administered. Studies evaluating nanoparticles effects on plasma coagulation time and their tendency to initiate vascular thrombosis are useful to assess nanoparticle thrombogenicity $[1,2,3]$.

A number of nanotechnology approaches have been borrowed and applied in stent technology. Stents with biofunctionalized surfaces with nanoparticles with high stability and carrier capacity, able to reduce the interaction with blood components and to incorporate substances as carriers for bioactive factors and drugs have been manufactured. There are already implants in the form of drug-eluting or biodegradable stents that induce healing reactions by triggering the body's natural processes $[2,3]$.

At present, there is not much data on the thrombogenicity of stent materials dictated by nanoscale observations. Because thrombogenicity is a multiparametric process, our study tests the hypothesis of the influence of surface nanotopography on platelet activation, in order to produce nano-coatings with less thrombogenic stent by adapting their surface properties. It is in fact intended to implement a real-time study of platelet response to biomaterials to improve hemocompatibility.

The primary phenomenon of blood-material interaction was the rapid and selective adsorption of proteins through a three-step process represented by the transport to the interface, the adsorption reaction and the conformational rearrangement. Thus, the engineering of the surface properties (physical and chemical characteristics) of the implants aims to reduce the adsorption of proteins and cellular interactions resulting in the improvement of implant biocompatibility.

Platelets are the main cells present in the blood that play the most important role in blood-material interactions. The adhesion of platelets to a surface is conditioned by two independent mechanisms. These are the transport of platelets to the surface, which depends on the flow conditions and the reaction of platelets with the surface, which depends on the nature of the surface and the adsorbed proteins.

In this paper, we want to verify the influence of nanotopography and surface characteristics on the thrombogenicity of different possible types of nanocoatings ( $\mathrm{ZnO}, \mathrm{TiO}_{2}$ and $\left.\mathrm{Fe} 3 \mathrm{O} 4\right)$. Thus, the results obtained by us are in line with previous hemocompatibility studies in the literature regarding the influence of these nanoparticles on coagulation. These lead us to the conclusion that nanotopography and surface roughness of biomaterials influence their biological behaviour. The studies within our project will be continued by thrombogenicity analyses on nanoparticle-coated metal bands.

This study about thrombogenicity induced by the nanoparticle is the subject of the grant: "New diagnostic and treatment methodologies: current challenges and technological solutions based on nanoparticles and biomaterials", that won the 2017 complex projects completed in consortia CDI, grant number: PN-III-P1-1.2-PCCDI-2017-0062, funded by CNCS - UEFISCDI Romania.

\section{REFERENCES}

1. Hansi, C., Arab, A., Rzany, A., Ahrens, I., Bode, C., \& Hehrlein, C. (2009). Differences of platelet adhesion and thrombus activation on amorphous silicon carbide, magnesium alloy, stainless steel, and cobalt chromium stent surfaces. Catheterization and Cardiovascular Interventions, 73(4), 488-496.

2. Karagkiozaki, V. C., Logothetidis, S. D., Kassavetis, S. N., \& Giannoglou, G. D. (2010). Nanomedicine for the reduction of the thrombogenicity of stent coatings. International journal of nanomedicine, 5, 239.

3. Krajewski, S., Neumann, B., Kurz, J., Perle, N., Avci-Adali, M., Cattaneo, G., \& Wendel, H. P. (2015). Preclinical evaluation of the thrombogenicity and endothelialization of bare metal and surface-coated neurovascular stents. American Journal of Neuroradiology, 36(1), 133-139.

4. Neun, B. W., \& Dobrovolskaia, M. A. (2011). Method for in vitro analysis of nanoparticle thrombogenic properties. In Characterization of nanoparticles intended for drug delivery (pp. 225-235). Humana Press.

5. Scott E. McNeil (ed.), Characterization of Nanoparticles Intended for Drug Delivery, Methods in Molecular Biology,cVol. 1682, DOI 10.1007/978-1-4939-7352-1_10, (c) Springer Science+Business Media LLC 2018;

6. Weber, M., Steinle, H., Golombek, S., Hann, L., Schlensak, C., Wendel, H. P., \& Avci-Adali, M. (2018). Blood-Contacting Biomaterials: In vitro evaluation of the hemocompatibility. Frontiers in Bioengineering and Biotechnology, 6.

7. Yang, J. Y., Bae, J., Jung, A., Park, S., Chung, S., Seok, J., ... \& Jeong, J. (2017). Surface functionalization-specific binding of coagulation factors by zinc oxide nanoparticles delays 
coagulation time and reduces thrombin generation potential in vitro. PloS one, 12(7), e0181634.

8. Xue, L. L., Long, P., Wei, H., \& Liang, Y. (2011). Hemocompatibility of $\mathrm{TiO} 2$ nanoparticles composite PTFE coating for medical devices. In Advanced Materials Research (Vol. 299, pp. 600-603). Trans Tech Publications. 